\title{
TRATAMIENTO DE REFLUJO VESICOURETERAL
}

\author{
Ribeiro de Castro, Pedro Massó y Armando Reis.
}

Servicio de Urología. Hospital María Pia. Porto. Portugal.

Resumen.- OBJETIVO: El reflujo vesicoureteral (RVU) es una patología frecuente, con una incidencia de 29/50\% en niños estudiados por infección del tracto urinario (ITU) y de 20\% en recién nacidos con diagnóstico pre-natal de hidronefrosis. A lo largo de los años, la terapéutica ha sido motivo de muchas reuniones, de muchos trabajos de investigación y continúa siendo un tema discutido. Con el desarrollo de técnicas endoscópicas de mínima invasión, por inyección sub-ureteral de "bulking agents", el grupo de candidatos a tratamiento quirúrgico aumentó. Presentamos los resultados del tratamiento quirúrgico de RVU de 2001 a 2006.

MÉTODOS: Se realizó un estudio retrospectivo de tratamiento endoscópico de RVU con inyección sub-ureteral de copolimero de dextranomero ácido hialurónico (Copol-Dx/AH). Se incluyeron todos los niños tratadas en el periodo comprendido entre 1/ Julio/ 2001 a 30/Diciembre/ 2006. El tratamiento se realizó en niños con un reflujo mínimo de grado II o superior. Se consideró tratados a los pacientes que presentaban una CUMS de control sin reflujo o con reflujo vesico ureteral grado I, proponiéndose en estos casos la suspensión de la profilaxis antibiótica de ITU.

RESULTADOS: Se trataron 661 niños, 607 por vía endoscopia y 54 por técnica de Cohen. De los niños tratados endoscopicamente, 437 eran de sexo femenino y 170 de sexo masculino. El RVU era bilateral en 37,7\% de los casos, siendo el grado Il el más frecuente $140 \%$ en el sexo masculino y $57 \%$ en el femenino). La tasa global de éxito fue de $70 \%$ después del primer tratamiento, de $75 \%$ después del segundo tratamiento y subió para $78 \%$ después del tercero.

CONCLUSIONES: La inyección sub-ureteral de copolímero de dextranomero ácido hialurónico es un tratamiento eficaz en niños con RVU, con indiferencia del grado de reflujo. Es un procedimiento sencillo, seguro, bien tolerado y con baja morbilidad. Es, en este momento, el tratamiento quirúrgico de elección en la mayor parte de los pacientes con RVU.

Palabras clave: Reflujo vesicoureteral. Tratamiento endoscópico de RVU.

Summary.- OBJECTIVES: Vesicoureteral reflux (VUR) is a frequent pathology, with an incidence of 29/50\% in children studied for urinary tract infection (UTI) and $20 \%$ of newborns with the diagnosis of prenatal hydronephrosis. Over the years, the treatment has been the subject of many meetings, many research studies, and continues being a topic under discussion. The number of candidates for surgical treatment increased with the development of minimally invasive endoscopic techniques by subureteral injection of bulking agents. We present the results of the surgical treatment of VUR between 2001 and 2006.

METHODS: We performed a retrospective study of the endoscopic treatment of VUR by subureteral injection of Dextranomer and hyaluronic acid copolymer (Copol-Dx/AH). All children
Ribeiro de Castro Servicio de Urología Hospital María Pia. Porto. (Portugal). armandobreis@hotmail.com lvis.joao.castro@gmail.com 
undergoing treatment between July 1 st 2001 and December 30th 2006 were included in this study. Treatment was performed in children with VUR grade II or greater. All patients presenting no reflux or grade I VUR on control VCUG were considered cured; stopping antibiotic prophylaxis was proposed in these cases.

RESULTS: 661 children underwent treatment, 607 endoscopic and 54 with the Cohen technique. Among children treated endoscopically, 437 where females and 170 males. VUR was bilateral in $37.7 \%$ of the cases, with grade II being the most frequent $140 \%$ in males and $57 \%$ in females). Overall success rate was 70\% after first treatment, 75\% after second treatment and increased to $78 \%$ after the third.

CONCLUSIONS: Subureteral injection of dextranomer and hyaluronic acid copolymer is an effective treatment in children with VUR, independently of the grade. It is a simple, safe, well tolerated procedure with low associated morbidity. Currently, it is the surgical treatment of choice in most patients with VUR.

Keywords: Vesicoureteral reflux. Endoscopic treatment.

\section{INTRODUCCIÓN}

El servicio de Urología del Hospital Maria Pia (H.M.Pia) es un servicio de referencia para la parte norte del país. Está constituido por un urólogo y un cirujano pediátrico. Dan formación de pos grado a los residentes de la especialidad de Urología y Cirugía Pediátrica de la gran mayoría de hospitales de la zona norte de Portugal. En el transcurso del año 2006 el servicio realizó 2.229 consultas, efectuó 548 cirugías, realizó 173 estudios de urodinamia y efectuó 793 CUMS. Los elementos del servicio realizan varias reuniones para discutir casos clínicos en los diversos servicios de Pediatría de los diferentes hospitales, eso permite orientar hacia nuestro servicio aquellos pacientes que requieren tratamiento quirúrgico, o un examen diagnóstico no disponible en el hospital de origen.

Como en cualquier servicio de urología pediátri$\mathrm{ca}$, una de las patologías más frecuentes es el RVU.

Durante muchos años, hasta el 2001, la técnica quirúrgica de elección fue la ureteroneocistostomía de Cohen. Desde ese año, la técnica más utilizada pasó a ser el tratamiento endoscópico con Copol-DX/AH (1-5).

El alto índice de resolución espontánea que se da en la propia historia natural de la enfermedad, continua siendo un aspecto importante cuando nos enfrentamos a un nuevo caso de RVU.

Continuamos defendiendo como criterios para tratamiento quirúrgico, los casos en que la profilaxis antibiótica no es eficaz o no se cumple, los casos con vejiga de baja compliance que no remiten al tratamiento médico, los RVU grado IV y grado V con historia de pielonefritis asociada, los casos con disminución en la función renal (DMSA), los casos con RVU persistente (superior a 5 años) - los casos de RVU con alteraciones de la unión uretero vesical (Ej. divertículo para ureteral) (6-7).

\section{MATERIAL Y MÉTODO}

Durante el periodo comprendido entre el 1 julio de 2001 y el 30 de diciembre de 2006 en nuestra institución se trató a 661 niños con RVU, de esos, a 54 se les aplicó la técnica de Cohen y a 607 la técnica endoscópica (Tabla l).

De los niños tratados com la técnica endoscópica $472(72 \%)$ son de sexo femenino y $170(28 \%)$ de sexo masculino (Tabla II y Figura 2).

La mayor parte de los niños pertenecían al grupo de edad comprendido entre los 5 y los 10 años $(56 \%)$, < de 1 año el $1 \%$, entre 1 y 5 años, $30 \%$, por encima de 10 años, $13 \%$ (Tabla III y Figura 3 ).

El RVU era bilateral en 229 niños $(37.7 \%)$, izquierdo en $39,3 \%$ y derecho en $23 \%$ (Tabla IV y Figura 4 ).

Todos los casos de RVU de grado I correspondían a RVU bilateral con RVU contra lateral de grado II o superior.

La mayor parte de los niños tenían RVU grado III y IV (Tabla $\vee$ y Figura 5).

\section{RESULTADOS}

A todos los niños se les realizó una CUMS de control (radiológica o isotópica).

Se consideró como éxito en el tratamiento, a los RVU de grado I que antes del tratamiento endoscópico eran de grado superior a I, ya que supuso la retirada de la profilaxis antibiótica.

La tasa global de éxito fue de $70 \%$ después del primer tratamiento, $75 \%$ después del segundo y de $78 \%$ después del tercer tratamiento. La eficacia del tratamiento en unidades con duplicidad uretero-pielo- calicial completa fue de $75 \%$.

TABLA I. NÚMERO TOTAL DE REFLUJOS. ACTITUD QUIRÚRGICA.

\begin{tabular}{|l|l|}
\hline Total $=$ & 661 \\
\hline Endoscópico & 607 \\
\hline Abierto & 54 \\
\hline
\end{tabular}


TABLA II.

\begin{tabular}{|l|l|l|}
\hline Masculino & 170 & $28 \%$ \\
\hline Femenino & 437 & $72 \%$ \\
\hline
\end{tabular}

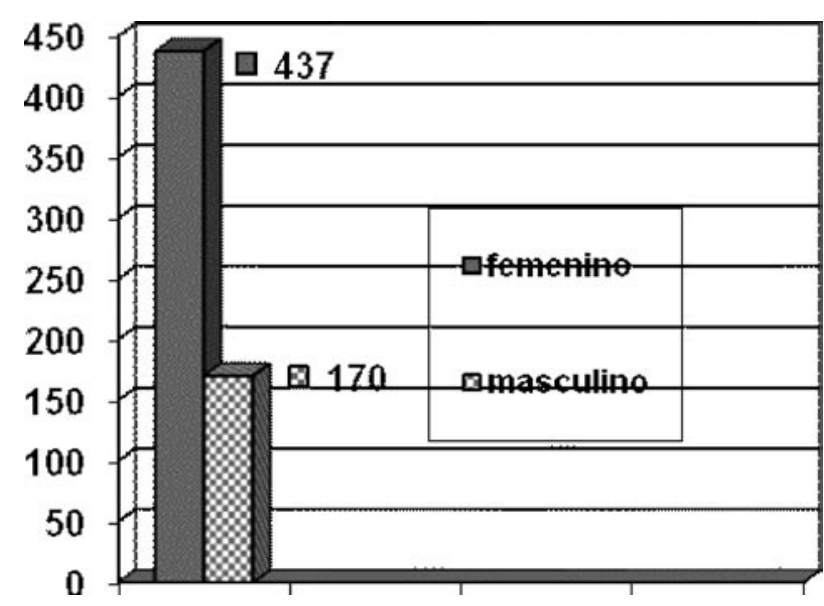

FIGURA 2. Distribución por sexo.

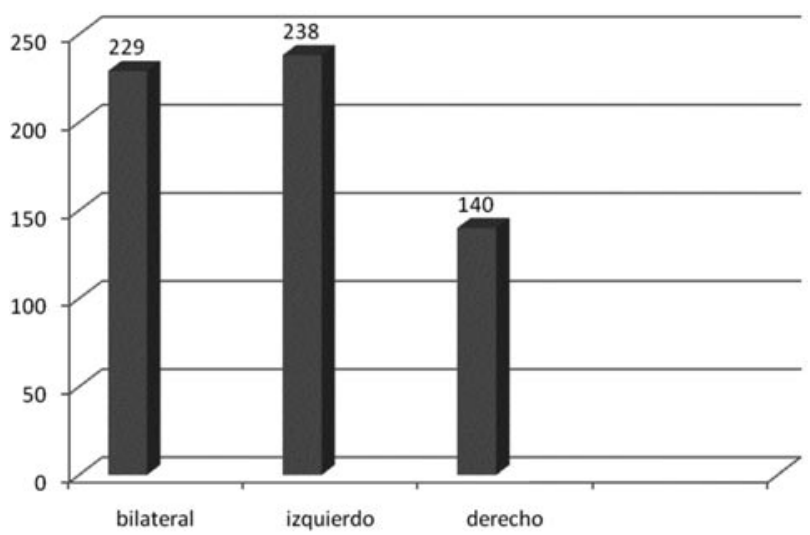

FIGURA 3. Tratamiento endoscópico: localización del reflujo.

TABLA III. TRATAMIENTO ENDOSCÓPICO.

\begin{tabular}{|l|l|l|}
\hline Bilateral & 229 & $37,7 \%$ \\
\hline Izquierdo & 238 & $31.3 \%$ \\
\hline Derecho & 140 & $23 \%$ \\
\hline
\end{tabular}

Con respecto a las complicaciones, tuvimos un caso de hemorragia intravesical que obligó a realizar una transfusión sanguínea y 8 pacientes con RVU post tratamiento contra lateral "de novo" en la CUMS de control.

\section{DISCUSIÓN}

La técnica anti-RVU clásica (ureteroneocistostomía de Cohen) tiene una tasa de curación de casi $100 \%$. A pesar de todo, obliga a realizar una cirugía abierta con una duración media de ingreso de 8 días. Continuamos defendiendo unos criterios bien definidos para el tratamiento quirúrgico, una vez que la propia historia natural de la enfermedad lleva a un elevado porcentaje de remisión espontánea del reflujo (8-11).

Todos los casos propuestos para intervención quirúrgica son discutidos con los pediatras que refieren a los pacientes. Los criterios de inclusión para tratamiento quirúrgico del servicio de urología son:

- Profilaxis antibiótica ineficaz.

- No cumplir la profilaxis antibiótica.

- Vejiga de baja compliance que no remite con tratamiento médico.

TABLA N.

\begin{tabular}{|c|c|c|}
\hline Grupo edad (años) & & $\mathrm{N}=607$ \\
\hline$<1$ & $1 \%$ & 6 \\
\hline $1-5$ & $30 \%$ & 182 \\
\hline$>5-10$ & $56 \%$ & 340 \\
\hline$>10$ & $13 \%$ & 79 \\
\hline
\end{tabular}

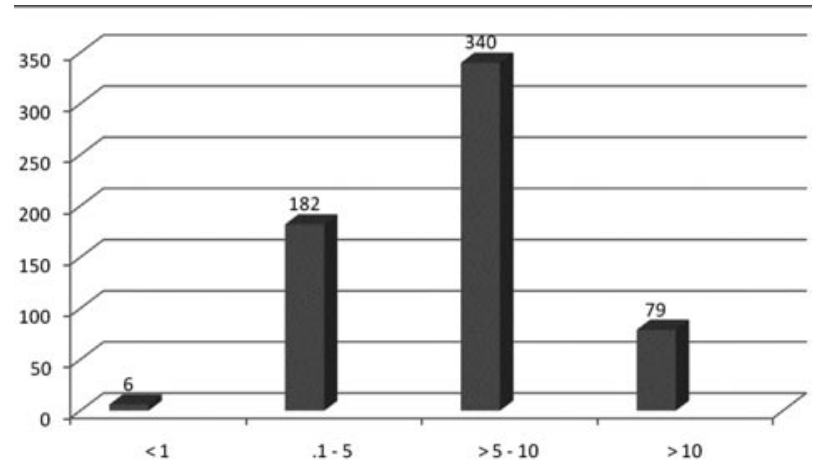

FIGURA 4. Distribución por grupo edad al momento del tratamiento. 


\begin{tabular}{|c|c|c|c|c|c|}
\hline & Grado I & Grado II & Grado III & Grado IV & Grado V \\
\hline Masculino & $9 \%$ & $21 \%$ & $40 \%$ & $24 \%$ & $6 \%$ \\
\hline Femenino & $8 \%$ & $19 \%$ & $57 \%$ & $15 \%$ & $1 \%$ \\
\hline
\end{tabular}

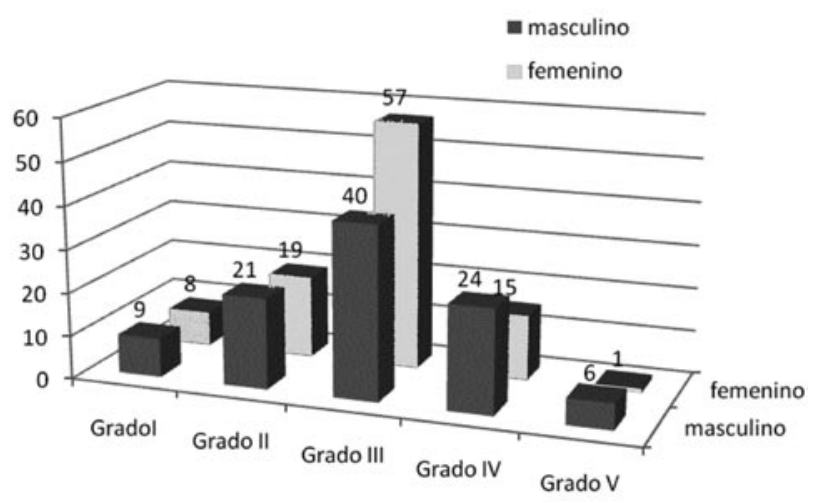

FIGURA 5. Distribución por sexo e grado de reflujo (\%).

- RVU de grado IV y $V$ con historia de pielonefritis.

- Disminución de la función renal (DMSA).

- RVU que perdura más allá de los 5 años.

- Asociación de RVU con alteraciones en la unión uretero vesical.

\section{CONCLUSIONES}

La inyección sub-ureteral de copolimero de dextranomero ácido hialurónico es un tratamiento efectivo en niños con RVU, indistintamente del grado de reflujo. Es un procedimiento simple, seguro, con buena tolerancia y con baja morbilidad. Es, en este momento, el tratamiento quirúrgico de elección en la gran mayoría de niños con RVU.

\section{BIBLIOGRAFÍA Y LECTURAS RECOMENDADAS} ("lectura de interés $y^{* *}$ lectura fundamental)

*1. CAPOZZA, N.; CAIONE, P.: "Role of the endoscopic treatment of vesicoureteral reflux. A 16-years experience". Minerva Pediatr., 55: 607, 2003.

*2. PURI, P.; CHERTIN, B.; VELAYUDHAM, M. y cols.: -Treatment of vesicoureteral reflux by endoscopic injection of dextranomer/hyaluronic Acid copolymer: Preliminary results". J. Urol., 170: 1541, 2003.

*3. PURI, P.; PIRKER, M.; MOHANAN, N. y cols.: "Subureteral dextranomer/hyaluronic acid injection as first line treatment in the management of high grade vesicoureteral reflux". J. Urol., 176: 1856, 2006.

*4. LÄCKGREN, G.; SKÖLDENBERG, E.; STENBERG, A.: "Endoscopic treatment with stabilized nonanimal hyaluronic acid/dextranomer gel is effective in vesicoureteral reflux associated with bladder dysfunction". J. Urol., 177: 1124, 2007.

*5. O'DONNELL, B.; PURI, P.: “Technical refinements in endoscopic correction of vesicoureteral reflux". J. Urol., 140: 1101, 1988.

*6. HIGHAM-KESSLER, J.; REINERT, S.E.; SNODGRASS, W.T. y cols.: "A review of failures of endoscopic treatment of vesicoureteral reflux with dextranomer microspheres". J. Urol., 177: 710, 2007.

*7. ZIVKOVIC, D.; VARGA, J.: "Endoscopic correction of vesicoureteric reflux-our thirteen years experience". Eur. J. Pediatr. Surg., 16: 245, 2006.

*8. CHERTIN, B.; PURI, P.: "Endoscopic management of vesicoureteral reflux: Does it stand the test of time?". Eur. Urol., 42: 598, 2002.

*9. MIYAKITA, H.; NINAN, G.K.; PURI, P.: "Endoscopic correction of vesico-ureteric reflux in duplex systems". Eur. Urol., 24: 111, 1993.

*10. JIMENEZ ALVAREZ, C.; ALAMINOS MINGORANCE, M.; MARTINEZ MARTINEZ, L.: "Scientific evidence in the endoscopic treatment of the vesico-ureteral reflux". Cir. Pediatr., 15: 122, 2002.

*11. LOPEZ LOPEZ, J.; SANCHEZ ZALABARDO, J.; SANCHEZ ELIPE, M. y cols.: "Endoscopic treatment of vesicoureteral reflux". An. Esp. Pediatr., 54: 132, 2001. 